\title{
The Form and Content of Cannabis Products in the United States
}

\author{
Stacey Steigerwald, MSSA ${ }^{7}$, Peggy O. Wong, $B A^{7}$, Arianne Khorasani, $B A^{2}$, \\ and Salomeh Keyhani, MD, MPH \\ ${ }^{1}$ NCIRE, San Francisco VA Medical Center, San Francisco, CA, USA; ${ }^{2}$ John Abbot College, Montreal, QC, Canada; ${ }^{3}$ Department of Medicine, \\ University of California at San Francisco, San Francisco, CA, USA; ${ }^{4}$ San Francisco VA, San Francisco, CA, USA.
}

J Gen Intern Med 33(9):1426-8

DOI: $10.1007 / \mathrm{s} 11606-018-4480-0$

(C) Society of General Internal Medicine (This is a U.S. Government work and not under copyright protection in the US; foreign copyright protection may apply) 2018

\section{INTRODUCTION}

Thirty states have legalized cannabis in some form. Cannabis potency is largely estimated from cannabis seized in drug raids and state-controlled producers. Little data exists about the tetrahydrocannabinol (THC) content and form of products commercially available.

Recreational states have defined potency thresholds in edibles, limiting THC to 50 or $100 \mathrm{mg}$ per package and 5 or $10 \mathrm{mg}$ per serving depending on the state. No clear limit exists on THC in other product forms. In addition, while recreational states prohibit false marketing claims and advertising aimed at children, no data exists on how well regulations are enforced. ${ }^{1}$

We collected data on form, THC concentration, and associated marketing claims of products in dispensaries to better understand products available and marketing targeted at the public.

\section{METHODS}

We identified the top three dispensaries with the most consumer reviews as a metric of public interest, reviewing a total 65 dispensaries. Six states and DC do not allow dispensaries. We extracted data on all products available for the most reviewed dispensary in each state ( 8 recreational states, 15 medical states). We determined sample representativeness by comparing our data with two more randomly selected dispensaries from the original list. When no new products were identified in form or THC content, data collection was considered complete. Information on form, marketing and nutritional claims, and THC content was collected for each product. Data was summarized by medians and interquartile range of THC content.

\section{RESULTS}

We identified 2264 unique products, 710 (31.4\%) in recreational states and $1546(68.3 \%)$ in medical states (Table 1).

Published online May 16, 2018
Among those, 1294 (57\%) listed THC by serving or package. In recreational states, THC content in edibles ranged from 5 to $7000 \mathrm{mg}$ among packages. About 4.2\% $(N=30)$ of packages exceeded the $100 \mathrm{mg}$ limit and $4.0 \%(N=28)$ of servings exceeded the $10 \mathrm{mg}$ limit. Among all edibles, $8.1 \%(n=183)$ were in forms potentially attractive to children (e.g., candy). In recreational states, median $\mathrm{THC}$ in extracts was $1000 \mathrm{mg}$ in packages and $100 \mathrm{mg}$ in servings, with $65.9 \%(N=81)$ of packages exceeding $100 \mathrm{mg}$ and $50.4 \%(N=62)$ of servings exceeding $10 \mathrm{mg}$.

Among all products, 473 (20.9\%) claimed therapeutic efficacy, $34 \%(N=160)$ of which were identified in recreational states. Most common was pain management ( $n=332,14.7 \%)$, anxiety $(n=188,8.3 \%)$, insomnia $(n=188,8.3 \%)$, depression $(n=156,6.9 \%)$, and stress $(n=117,5.2 \%)$. Other indications (e.g., seizures, PTSD) were less frequent $(n=28,1.3 \%)$. Fifteen $(0.7 \%)$ made nutritional claims (e.g., "vitamin E", "gluten-free, vegan", “omega 3 "). Nine (0.4\%) warned of adverse effects (e.g., dry mouth).

\section{DISCUSSION}

Most edible products adhered to THC limits. However, the regulatory vacuum constraining $\mathrm{THC}$ content in other products has resulted in a proliferation of cannabis with high $\mathrm{THC}$ concentrations. We also found over $20 \%$ of products claimed therapeutic efficacy and 1 out of 12 edibles was potentially appealing to children.

The development of new products with high THC concentrations has outpaced safety data. A pooled analysis of 79 trials examining the efficacy of cannabis for several conditions reported severe adverse events including vomiting, diarrhea, disorientation, anxiety, confusion, dyspnea, seizures, psychosis, hallucination, and paranoia. ${ }^{2}$ The median dose of THC in cannabinoid pharmaceuticals in these trials was $8 \mathrm{mg}$. High potency cannabis has also been linked to psychosis, rapid onset of paranoid delusions, and catatonia. ${ }^{3}$ These data raise concerns given the high THC content available in some recreational products.

We found $20 \%$ of products advertised therapeutic benefits. Benefits attributed to cannabis included conditions where there is limited data available or conditions that cannabis may worsen like anxiety and depression, ${ }^{4,5}$ suggesting regulations are inadequately enforced. No regulations currently 
Table 1 THC Levels Listed for the Cannabis Products with Reported THC Content $(N=\mathbf{2 2 6 4})$

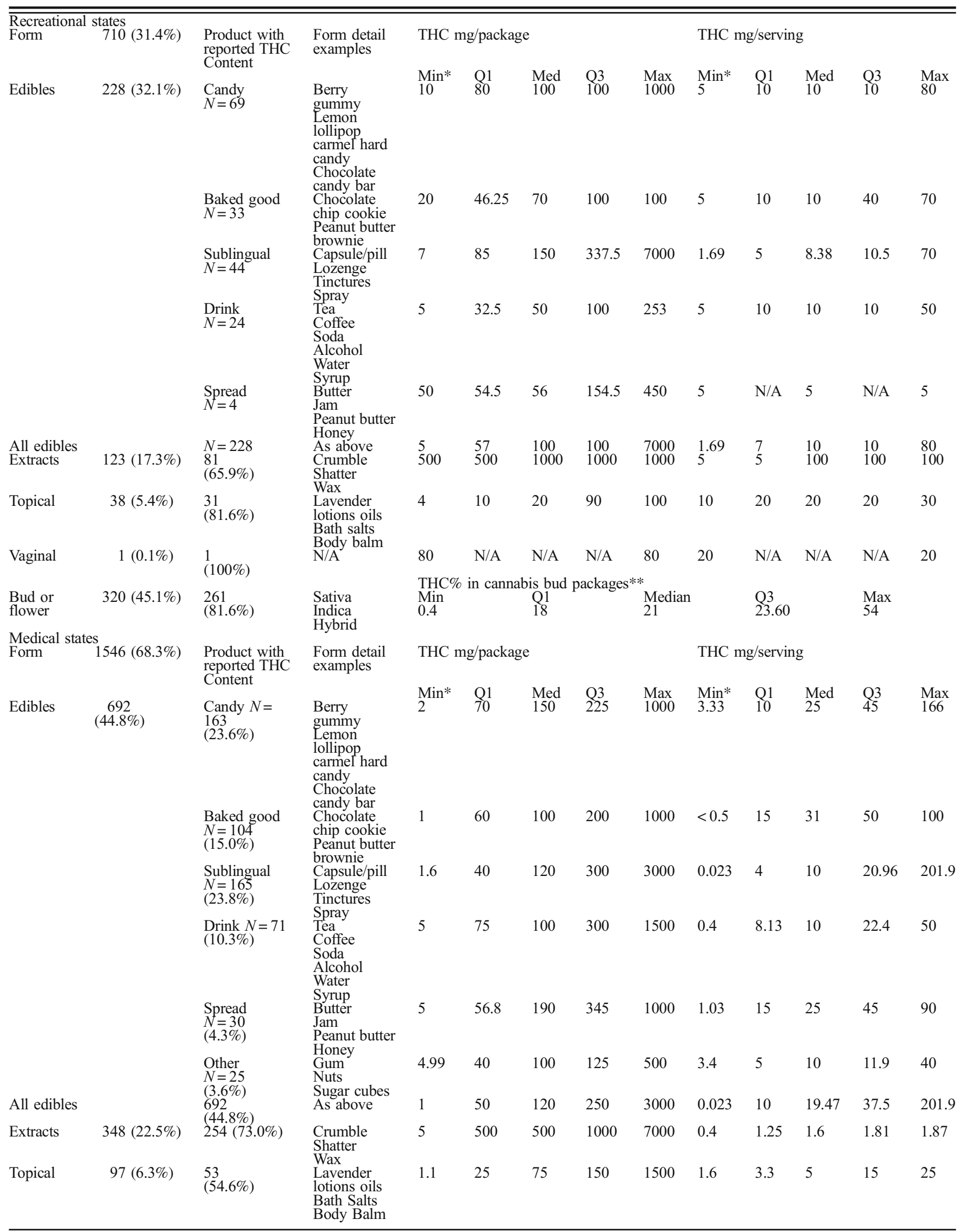


Table 1. (continued)

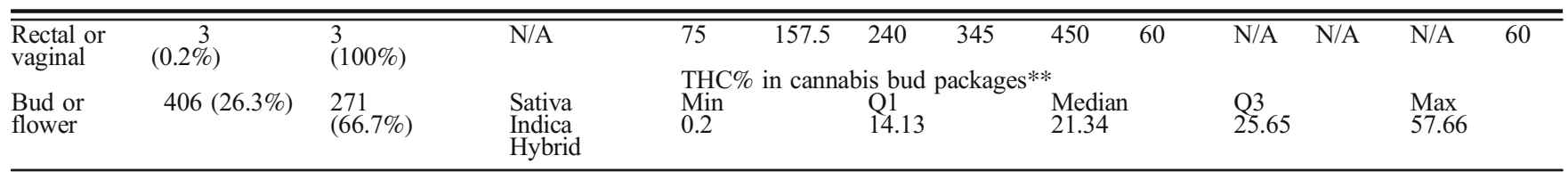

*Some products were Cannabidiol only products with minimal THC content

**THC is reported by percentage of weight only in bud/flower products

constrain product form or flavor and many edibles are in forms potentially appealing to children (e.g., candy, flavored drinks). This is of concern given the rise in pediatric poisoning with cannabis products. ${ }^{6}$

Our methods have several limitations. In absence of sales data, consumer reviews were used as a metric of dispensary interest. We also used websites to identify products and all products may not have been listed. Finally, some products did not provide THC content. While the sampling strategy had some limitations, it nonetheless provides a clear picture of products available to the public.

In conclusion, the proliferation of high THC cannabis products combined with inadequate marketing oversight may pose risks to public health. More regulatory oversight is needed.

Corresponding Author: Stacey Steigerwald, MSSA; NCIRE, San Francisco VA Medical Center, San Francisco, CA, USA (e-mail: Stacey. Steigerwald@va.gov).

\section{Compliance with Ethical Standards:}

Conflict of Interest: The authors whose names are listed in this publication certify that they have NO affiliations with or involvement in any organization or entity with any financial interest (such as honoraria; educational grants; participation in speakers' bureaus; membership; employment consultancies, stock ownership, or other equity interest; and expert testimony or patent-licensing arrangements), or non-financial interest (such as personal or professional relationships, affiliations, knowledge or beliefs) in the subject matter or materials discussed in this manuscript.

\section{REFERENCES}

1. Hibpshman D. Marijuana licensing updates [PowerPoint Slides]. 2017. Retrieved from http://www.oregon.gov/olcc/marijuana/Pages/default. aspx.

2. Whiting PF, Wolff RF, Deshpande S, et al. Cannabinoids for medical use: a systematic review and meta-analysis. JAMA. 2015;313(24):2456-73.

3. Di Forti M, Marconi A, Carra E, et al. Proportion of patients in south London with first-episode psychosis attributable to use of high potency cannabis: a case-control study. Lancet Psychiatry. 2015;2(3):233-8.

4. Hasin DS. US epidemiology of cannabis use and associated problems. Neuropsychopharmacology. 2018;43(1):195-212.

5. Nugent SM, Morasco BJ, O’Neil ME, Freeman M, Low A, Kondo K, et al. The effects of cannabis among adults with chronic pain and an overview of general harms: a systematic review. Ann Intern Med. 2017;167:319-31. doi:https://doi.org/10.7326/M17-0155.

6. Wang GS, et al. Unintentional pediatric exposures to Marijuana in Colorado, 2009-2015. JAMA Pediatr. 2016;170(9):e160971. 